\title{
INDICADORES DE SUSTENTABILIDAD EN EL ECOTURISMO INDÍGENA DE MÉXICO
}

\section{INDICATORS OF SUSTAINABILITY IN THE INDIGENOUS ECOTOURISM OF MEXICO}

\author{
Rosa María Velázquez-Sánchez, ${ }^{1^{*}}$ \\ Joao Gabriel Boto-De Matos Caeiro, Ri Ricardo Alarcón-Alcántara, ${ }^{3}$ \\ Jesús Gómez-Velázquez ${ }^{4}$ \\ Fecha de recepción: 31 de enero de 2018 \\ Fecha de aceptación: 2 de abril de 2018
}

\begin{abstract}
Resumen - Se planteó realizar un estudio de los indicadores de sustentabilidad en las comunidades indígenas con ecoturismo, el cual se llevó a cabo en los estados de Chiapas, Veracruz, Campeche, Yucatán, Guerrero, Michoacán y Oaxaca. Se colectó la información necesaria para identificar y definir las categorías principales de sustentabilidad. Para examinar los datos del cuestionario piloto se empleó el análisis factorial a componentes principales; el procedimiento para la prueba de validez del cuestionario proporcionó los elementos para depurar el instrumento con el que se recabaría la información, lo que se supeditó a las condiciones de acceso, pero sobre todo a la situación de inseguridad y conflictos locales que presentaron las comunidades incluidas en el estudio. Los resultados permitieron definir los componentes de los indicadores que ayudan a conceptualizar la sustentabilidad en las comunidades indígenas con ecoturismo.
\end{abstract}

\section{Palabras clave:}

Sustentabilidad, turismo, comunidades, ecoturismo indígena.
Abstract - It was proposed to conduct a study on sustainability indicators in indigenous communities with ecotourism. This study was conducted in the mexican states of Chiapas, Veracruz, Campeche, Yucatan, Guerrero, Michoacán and Oaxaca. For data analysis of the pilot questionnaire, the factorial analysis to main components was used. The validity test of the questionnaire provided the elements for debugging the instrument for definite data collection. This was subject to access conditions, but mainly to the insecurity situation and local conflicts presented by the communities contemplated in this study. The results allowed defining the components of the indicators that help to conceptualize the sustainability in the indigenous communities with ecotourism.

\section{Keywords:}

Sustainability, tourism, communities, indigenous ecotourism.

\footnotetext{
${ }^{1}$ Facultad de Contaduría y Administración de la Universidad Autónoma "Benito Juárez" de Oaxaca. Correo electrónico: romavesa205@yahoo.com.mx 2 Facultad de Bellas Artes de la Universidad Autónoma "Benito Juárez" de Oaxaca. Correo electrónico: caeirojoao@gmail.com 


\section{Introducción}

1 nivel mundial, el turismo representa 4 una de las actividades económicas con 1 mayor competencia. De acuerdo con datos recopilados por la Secretaría de Turismo (Sectur, 2017), a través del Análisis Integral del Turismo (Datatur) de abril de 2017:

El Banco de México reportó que la llegada de viajeros internacionales para el primer cuatrimestre de 2017 fue de 32.5 millones, esto es, 727 mil viajeros más de los que lo hicieron en el mismo lapso del año pasado, lo que representó un crecimiento anual de $2.3 \%$, lo que equivale a 7 mil 605 millones de dólares y representa un incremento de $10.6 \%$ con respecto al mismo periodo de 2016 (Sectur, 2017).

Los resultados han permitido comprobar que el turismo representa para México una de las principales actividades generadoras de empleo y de divisas. Por su parte, el segmento de turismo alternativo es especializado; sin embargo, crece en oferta porque requiere menos inversión en infraestructura, pero más desarrollo del servicio. Constituye la opción para disfrutar de diversas actividades que demandan en su mayoría ambientes naturales y contacto con los habitantes locales; no obstante, no en todos los casos se le da seguimiento y muchas veces ocasiona deterioro ambiental y alteración en la forma tradicional de convivencia de las comunidades.

El ecoturismo es una variable del turismo alternativo que se define como "forma de turismo que ofrece al visitante disfrutar y apreciar atractivos naturales y culturales, a través de la promoción de la conservación del medio" (Organización Internacional del Trabajo y Organización Mundial del Turismo, OIT-UNWTO, 2014). Empero, el ecoturismo es una variante que puede representar el concepto de turismo sustentable, siempre y cuando se cumpla con dos aspectos esenciales: cuidado del medio natural y respeto por la cultura. Al revisar los criterios para el otorgamiento de la categoría de ecoturismo, se observa que en su mayoría no contemplan aspectos culturales ni medidas para conservar el medio natural.

Al respecto, la Comisión Nacional para el Desarrollo de los Pueblos Indígenas (CDI) administra un programa que impulsa el ecoturismo en comunidades indígenas, por medio de apoyos para la construcción de cabañas. La CDI tenía registrados 86 destinos con ecoturismo a nivel nacional, en 16 localidades indígenas (CDI, 2015). Al examinar las condiciones de los servicios prestados y las medidas para conservar los espacios naturales y la participación de los habitantes de las comunidades, se advirtió que la categoría de ecoturismo no corresponde a muchos de los prestadores de servicios.

El ideal que el ecoturismo pretende es la participación de los habitantes de la comunidad en la planeación y distribución de los beneficios para el progreso económico local (OIT-UNWTO, 2014), por lo que se esperaría que a través de la ejecución de proyectos de ecoturismo se lograra incorporar a los pobladores al desarrollo y que se observaran cambios positivos en sus condiciones de vida con el mínimo impacto a la naturaleza.

La realidad es que en México existen problemas para observar el efecto del ecoturismo en las comunidades debido a la competencia desleal, compuesta por un sinnúmero de destinos que operan con la denominación de ecoturismo sin serlo, ya que se dedican al aprovechamiento del medio natural, provocan deterioro, no incluyen la cultura y no cuentan con la participación de los habitantes de las comunidades, contrario a lo que pudiese entenderse como turismo sustentable.

La ausencia de mecanismos que regulen el ecoturismo en nuestro país ocasiona que se oferte este servicio sin serlo, con el consecuente desencanto del visitante, pero principalmente con un efecto negativo en la sustentabilidad de las comunidades indígenas en las que se alberga. Una de las causas principales es la falta de acciones que verifiquen las actividades para la convivencia y respeto con las formas de organización y cosmovisión del pueblo originario. En algunos de los casos observados, los aspectos culturales no se incluyen o se 
malinterpretan, porque se emplean como un objeto que integra el producto del turismo, con los efectos negativos para los pobladores (Velázquez y Gómez, 2017).

De acuerdo con Niño y Saldaña (2014), el falso "ecoturismo" ha ocasionado el desplazamiento de habitantes de comunidades originarias de Michoacán, Guerrero y Oaxaca, debido a la alteración del medio natural, la pérdida de identidad cultural y los efectos negativos en las condiciones de vida. Por lo anterior, es necesario diseñar mecanismos que permitan evaluar el impacto del ecoturismo en los sectores ambiental, cultural y económico de las poblaciones indígenas mexicanas para regular y certificar las actividades y servicios que se ofrecen. En este estudio contribuimos con la definición de los indicadores de sustentabilidad encontrados en el ecoturismo indígena.

\section{Revisión de literatura}

En Australia y Nueva Zelanda se ha registrado un significativo impulso a la conservación ambiental, a la protección de la biodiversidad, a la reglamentación para visitantes y a la vigilancia de la actividad turística. Se puede observar en estos países el avance en términos de destinos turísticos sustentables, gracias a la atención al cumplimiento de los estándares ambientales y a una valoración de la cultura. Al respecto, Finlandia ha abierto una alternativa con la zona de comunidades indígenas de los soami, en la que se permite la aproximación de visitantes a sus actividades culturales.

En los ejemplos de turismo sustentable certificado se puede constatar la importancia de orientar la prestación de servicios hacia la conservación del ambiente y la promoción de la cultura. El turismo sustentable prueba que evaluar y regular las tareas que se emprendan para impulsar la certificación y cumplimiento de estándares internacionales se refleja en indicadores económicos y contribuye a la competitividad del destino; sin embargo, no se considera valorar la inclusión de los habitantes de las comunidades en las que se encuentran los paisajes naturales.
En México se cuenta con un destino turístico certificado por un organismo internacional: las Bahías de Huatulco, empero, la entidad evaluadora no proporciona información para conocer los indicadores y el cumplimiento de estándares que emplea para otorgar la certificación y es evidente que sólo contempla aspectos ambientales, dejando a un lado la cultura y la participación de los nativos.

La información disponible sobre la actividad turística en México se localiza en informes como el de Datatur (2014). Los reportes oficiales detallan la importancia económica del turismo, en términos de generación de visitas y de empleos creados. En los reportes de la Sectur (2015) no se especifica el turismo alternativo. Del ecoturismo se conocen datos por algunos informes académicos como el realizado por la Sectur, en los que se analiza este sector y la importancia del ecoturismo para las comunidades indígenas. La CDI (2015), a través de su página oficial, facilita localizar los destinos y promociona los aspectos del ambiente y los elementos culturales.

En la literatura disponible se pueden consultar varios análisis sobre el ecoturismo, los cuales se han encargado de identificar y definir los indicadores que pueden dar cuenta del turismo sustentable en comunidades mexicanas. En este sentido, Velázquez et al. (2016) y Velázquez y Gómez (2017) han estudiado la sustentabilidad en los destinos ecoturísticos localizados en poblaciones indígenas.

Los resultados obtenidos permitieron establecer como hipótesis que sí es posible encontrar indicadores de sustentabilidad para proyectos de ecoturismo en comunidades indígenas, por lo que este trabajo se propuso proveer de información a fin de determinar dichos indicadores de sustentabilidad en el ecoturismo indígena, como un intento de revisar la definición del turismo sustentable.

Objetivo general: analizar elementos culturales para definir los indicadores de sustentabilidad en el ecoturismo indígena. 


\section{Metodología}

El estudio fue una combinación de análisis cualitativo con herramientas de fenomenología y de análisis cuantitativo. Se llevó a cabo a través de un procedimiento que contempló dos métodos de investigación orientados hacia la consolidación de una metodología para identificar categorías y definir indicadores de sustentabilidad en el ecoturismo en comunidades indígenas de Michoacán, Campeche, Veracruz, Guerrero, Chiapas, Yucatán y Oaxaca.

\section{Procedimiento}

Definir categorías como cultura representó una etapa a corto plazo en la que se vigiló el proceso de transformación de las categorías cualitativas a indicadores cuantitativos. La fase cualitativa tuvo una duración de cinco meses, debido a la complejidad del procedimiento, pero principalmente a la situación de desconfianza e inseguridad que se percibe en algunas comunidades. El proceso abarcó seis meses, en el que se entrevistó a profundidad a los informantes clave.
El procesamiento de la información para el análisis cualitativo se efectuó con apoyo del software atlas.ti, a través del cual se identificaron y definieron las categorías principales de turismo alternativo.

Los resultados cualitativos se definieron como la información empírica, en los datos obtenidos, la contextualización del fenómeno y en la aplicación de la encuesta. La base estructurada de datos con las categorías e indicadores permitió integrar el cuestionario con los indicadores de sustentabilidad definidos a partir de la cosmovisión de los habitantes de las comunidades indígenas con ecoturismo en las que se desarrolló el estudio.

Se llevó a cabo un análisis de corte cualitativo basado en los elementos de la fenomenología, para identificar y definir las categorías que conceptualizan la cosmovisión de los habitantes de las comunidades indígenas respecto de la cultura, a través del acercamiento a los informantes clave señalados por la comunidad con una entrevista profunda. Los resultados se muestran en la tabla 1.

Tabla 1.

Categorías de los elementos de cultura identificados en comunidades indígenas con ecoturismo en México

\begin{tabular}{|c|c|c|}
\hline VARIABLE & CATEGORÍAS & INDICADORES \\
\hline \multirow{3}{*}{ Sustentabilidad } & Cultura propia & $\begin{array}{l}\text { - Conocimiento tradicional } \\
\text { - Organización } \\
\text { - Normatividad } \\
\text { - Medicina tradicional } \\
\text { - Comida tradicional } \\
\text { - Usos y costumbres }\end{array}$ \\
\hline & Adecuación & $\begin{array}{l}\text { - Incorporación de cargos } \\
\text { - Incorporación de elementos modernos a la cocina tradicional } \\
\text { - Adecuación de la medicina tradicional } \\
\text { - Adecuación de la normatividad }\end{array}$ \\
\hline & Cosmovisión & $\begin{array}{l}\text { - Valores } \\
\text { - Tradiciones } \\
\text { - Fiestas } \\
\text { - Relación con la tierra } \\
\text { - Pertenencia }\end{array}$ \\
\hline
\end{tabular}

Se aprecian las categorías e indicadores de la variable sustentabilidad que fueron identificados y que derivaron de las entrevistas a profundidad con informantes clave en las comunidades de Campeche, Chiapas, Veracruz, Guerrero, Yucatán, Michoacán y Oaxaca. Fuente: elaboración propia. 
Con base en las categorías identificadas en el proceso de análisis cualitativo se construyó un cuadro operacional con los indicadores, con la finalidad de diseñar ítems y estructurar un cuestionario con 25 preguntas. Dicho cuestionario se probó como piloto en 25 sujetos, cuyo trabajo se relaciona con actividades de ecoturismo en poblaciones indígenas. Los datos se examinaron para la prueba de validez del cuestionario, por medio del análisis factorial a componentes principales.
Los resultados mostraron inconsistencia en ocho ítems, por lo que se procedió a revisar la redacción y una segunda intervención con los informantes clave. Los hallazgos de la prueba piloto permitieron obtener la varianza acumulada y estimar el tamaño de la muestra y el diseño con que se aplicó el cuestionario, lo que se observa en la tabla 2.

Tabla 2.

Diseño de la muestra de habitantes de las comunidades indígenas con ecoturismo en los estados de Campeche, Chiapas, Guerrero, Michoacán, Oaxaca y Veracruz, México

\begin{tabular}{|c|c|c|}
\hline ESTADO & DESTINOS DE ECOTURISMO & CUESTIONARIOS APLICADOS \\
\hline Campeche & 2 & 12 \\
\hline Chiapas & 5 & 10 \\
\hline Michoacán & 5 & 5 \\
\hline Guerrero & 2 & 16 \\
\hline Oaxaca & 6 & 10 \\
\hline Veracruz & 5 & 10 \\
\hline Yucatán & 4 & $\mathbf{6 9}$ \\
\hline TOTAL & $\mathbf{2 9}$ & \\
\hline
\end{tabular}

Se pueden observar los datos de los destinos ecoturísticos incluidos en el estudio por estado y por el número de entrevistados y cuestionarios aplicados. Cabe mencionar que debido a la situación de inseguridad y al complicado acceso a algunas de las comunidades fue difícil que los habitantes y visitantes mostraran disposición para responder a los cuestionarios, por lo que la muestra se convirtió a conveniencia. Fuente: elaboración propia.

Las entidades donde se aplicó el cuestionario fueron en Campeche: Servicios Ecoturísticos Caray S.C y Organización Ecoturismo Calakmul; Chiapas: Cascada del Chiflón Tzinol, Cascada del Chiflón Velo de novia, Causas Verdes las Nubes, Cascadas de Misol Ha y Bajilum Pakal; Michoacán: Pantzingo, Parador Turístico Marvata, Centro Ecoturístico Terutzkua, Parador Turístico Tata Vasco e Isla de Pacandai; Oaxaca: Ecoturismo Capulalpam, Ecoturismo Benito Juárez, Vega del Solzuzul, Ecoturismo Yaa Cuetzi, Ecoturismo Latuvi y Centro de Ecoturismo Santa
Catarina Lachatao; Veracruz: Centro Ecoturístico Montaña Okotentlu, Reserva Ecológica Jem Taaxi, Cascadas Encantadas, Mangle Rop y Centro Ecoturístico Ruta del Cangrejo; Yucatán: Centro Ecoturístico Cabañas Ecológicas, Uh Najil Ex, Balam AC y Cenote Ecoturístico X-Canches; Guerrero: Centro Ecoturístico Papalutla y Centro Ecoturístico La Montaña. Para confirmar la existencia de los destinos se acudió a las delegaciones de la Comisión para el Desarrollo de los Pueblos Indígenas en cada uno de los estados. 


\section{Resultados}

Como ya se mencionó, la etapa de análisis cuantitativo consistió en una fase de investigación que inició en cuanto se obtuvieron los datos cualitativos en términos de categorías. Se definieron indicadores de cada una de estas categorías para estructurar un cuestionario a través del cual se evaluarían tres variables.

Se aplicó un cuestionario piloto y se procedió a rectificarlo con la ayuda de expertos. Con los resultados de la prueba se practicó el análisis factorial para la validez correspondiente del instrumento y la estimación de la varianza. Con el cálculo y diseño de la muestra se aplicó el cuestionario a 25 participantes y se realizó la prueba de confiabilidad, la cual dio como resultado un alfa de Cronbach de 85.

En la tabla 3 se muestran los resultados. Sustentabilidad con las categorías: Cultura propia con seis indicadores; Adecuación cuatro indicadores y Cosmovisión cinco indicadores. Las respuestas reflejaron la validación de 15 indicadores para las categorías de la variable Sustentabilidad.

\section{Tabla 3.}

Categorías e indicadores de sustentabilidad en el ecoturismo de comunidades indígenas de México

\begin{tabular}{|c|c|c|c|}
\hline CATEGORIAS & INDICADORES & MEDIAS & $\begin{array}{c}\text { DESVIACIÓN } \\
\text { ESTÁNDAR }\end{array}$ \\
\hline \multirow{6}{*}{ Cultura propia } & Conocimiento tradicional (6) & 4.94 & 0.723 \\
\hline & Organización & 4.65 & 0.821 \\
\hline & Normatividad & 4.25 & 0.478 \\
\hline & Medicina tradicional & 3.60 & 0.378 \\
\hline & Comida tradicional & 4.16 & 0.213 \\
\hline & Usos y costumbres & 3.18 & 0.854 \\
\hline \multirow{4}{*}{ Adecuación } & Incorporación de cargos (4) & 3.23 & 0.742 \\
\hline & Incorporación de elementos modernos a la cocina tradicional & 4.39 & 0.638 \\
\hline & Adecuación de la medicina tradicional & 3.28 & 0.825 \\
\hline & Adecuación de la normatividad & 3.59 & 0.369 \\
\hline \multirow{5}{*}{ Cosmovisión } & Valores (5) & 4.29 & 0.259 \\
\hline & Tradiciones & 3.89 & 0.358 \\
\hline & Fiestas & 4.59 & 0.259 \\
\hline & Relación con la tierra & 4.69 & 0.356 \\
\hline & Pertenencia & 4.89 & 0.368 \\
\hline
\end{tabular}

En esta tabla se muestran los resultados del análisis de los 15 indicadores de las categorías que definen la sustentabilidad y que, a su vez, permiten un acercamiento a un constructo en las comunidades indígenas con ecoturismo. Fuente: elaboración propia. 
Como se puede observar en los resultados, la categoría Adecuación es un descubrimiento entre las comunidades como un elemento para permanecer con sus estructuras tradicionales, pero con la forma de resistencia a la que hace mención Martínez (2013). Con los resultados obtenidos se examinó cada uno de los centros ecoturísticos incluidos en el estudio, para determinar con base en la presencia de los indicadores un nivel de sustentabilidad como medio comparativo por el tipo de participación o presencia de los habitantes en la prestación y administración de los puntos de ecoturismo. En la tabla 4 se presenta un concentrado de los lugares representativos de cada una de las categorías observadas.

Tabla 4.

Indicadores por categorías de sustentabilidad en el ecoturismo de comunidades indígenas de México

\begin{tabular}{|c|c|c|c|c|}
\hline DESTINO ECOTURÍSTICO & $\begin{array}{l}\text { VARIABLE / } \\
\text { INDICADORES }\end{array}$ & SUMA & $\%$ & ÍNDICE \\
\hline \multirow{3}{*}{$\begin{array}{l}\text { I. Servicios Ecoturísticos Caray } \\
\text { (Campeche) }\end{array}$} & Cultura propia 2 & 10 & 66 & Medio \\
\hline & Adecuación 4 & & & \\
\hline & Cosmovisión 4 & & & \\
\hline \multirow{3}{*}{ II. Cascadas de Misol Ha (Chiapas) } & Cultura propia 3 & 9 & 60 & Medio \\
\hline & Adecuación 4 & & & \\
\hline & Cosmovisión 2 & & & \\
\hline \multirow{3}{*}{$\begin{array}{l}\text { III. Centro Ecoturístico Teritzikua } \\
\text { (Michoacán) }\end{array}$} & Cultura propia 4 & 12 & 80 & Alto \\
\hline & Adecuación 3 & & & \\
\hline & Cosmovisión 5 & & & \\
\hline \multirow{3}{*}{ IV. Ecoturismo Capulalpam (Oaxaca) } & Cultura propia 5 & 14 & 93 & Alto \\
\hline & Adecuación 4 & & & \\
\hline & Cosmovisión 5 & & & \\
\hline \multirow{3}{*}{$\begin{array}{l}\text { V. Reserva Ecológica Jem Taaxi } \\
\text { (Veracruz) }\end{array}$} & Cultura propia 2 & 10 & 66 & Medio \\
\hline & Adecuación 4 & & & \\
\hline & Cosmovisión 4 & & & \\
\hline \multirow{3}{*}{$\begin{array}{l}\text { VI. Balam AC } \\
\text { (Yucatán) }\end{array}$} & Cultura propia 4 & 12 & 80 & Alto \\
\hline & Adecuación 4 & & & \\
\hline & Cosmovisión 4 & & & \\
\hline \multirow{3}{*}{$\begin{array}{l}\text { VII. Centro Ecoturístico Papalutla } \\
\text { (Guerrero) }\end{array}$} & Cultura propia 4 & 9 & 60 & Medio \\
\hline & Adecuación 4 & & & \\
\hline & Cosmovisión 1 & & & \\
\hline
\end{tabular}

En la tabla se ofrecen los resultados del análisis de los 15 indicadores de las categorías de sustentabilidad. Se muestra por categoría e indicadores con los porcentajes y el índice que determinó el procedimiento de evaluación. Los resultados proponen la estructura de una metodología para evaluar los destinos ecoturísticos sustentables. Fuente: elaboración propia. 
El procedimiento empleado en esta investigación permitió establecer y probar los indicadores para cada una de las categorías de sustentabilidad observadas en los destinos ecoturísticos que se localizan en comunidades indígenas. Como se mostró en los resultados, existen algunos que no cumplen con elementos de sustentabilidad y son precisamente los que no consideran la participación de los habitantes en las actividades y en la administración de los servicios.

La metodología empleada en este estudio es una propuesta para basar el análisis en elementos de cosmovisión que permiten el acercamiento a resultados con validez y confiabilidad. Con los procedimientos probados en esta investigación se propone un nuevo criterio para evaluar el turismo sustentable. Se observa que éste no puede ser clasificado como tal si no cumple con el cuidado del ambiente, pero también con resultados visibles para la participación de los habitantes de las comunidades, principalmente en la preservación de la cultura ancestral. En este estudio se contempla una nueva definición del ecoturismo en poblaciones indígenas que permita ofrecer al visitante "turismo sustentable", que cumpla con los tres grandes rubros de la sustentabilidad:

- Cuidado del medio natural

- Respeto y conservación a los aspectos culturales

- Vigilar que los beneficios generados del servicio ecoturístico recaigan en los habitantes de las comunidades en las que se ofrece.

Al supervisar el cumplimiento de los requisitos para el turismo sustentable y en particular, del ecoturismo en comunidades indígenas, se contribuye a conservar el ambiente y la presencia de la cultura de forma natural, con la asistencia y participación de sus pobladores.

\section{Conclusiones}

Como lo evidenciaron los resultados, se cumplió con el objetivo general, se analizaron los indicadores de sustentabilidad presentes en el ecoturismo de comunidades indígenas en México. Con dichos resultados se propone la metodología establecida para elaborar un diagnóstico que evalúe el ecoturismo en esos lugares.

Asimismo, los resultados mostraron que la sustentabilidad comunitaria se define por su cultura propia y su cosmovisión, pero que también es una forma de resistencia que, a través de la adecuación de elementos modernos, permite la permanencia de lo propio ante la modernidad que llega a las comunidades, situación que se pudo observar en el ecoturismo. Al respecto, es importante valorar la comunalidad como la expresó Díaz (2005) y que ha retomado Martínez (2013) para explicarla junto con el desarrollo.

La resistencia ya no se puede definir solamente como la espiral que describe Guerrero (2015), ahora es una adecuación a la corriente y a la velocidad del desarrollo, por lo que es sumamente importante analizar la permanencia de las comunidades indígenas en un proceso de cambio ante la globalización.

Otro aspecto relevante es la coincidencia con los resultados de Kiss (2004), al confirmar que las comunidades indígenas que se involucran en el ecoturismo por sí mismas conservan el ambiente como parte de su expresión cultural, y con los de Laverack y Thangphet (2007), quienes han demostrado la capacidad de las comunidades para participar en la administación del ecoturismo.

Acerca de los resultados previos recogidos en los estudios de Velázquez y Gómez (2017), se pueden comparar en términos de evolución de los indicadores y en las características particulares que se manifiestan en cada localidad, por lo que es posible proponer una metodología que permita generalizar el procedimiento hacia comunidades indígenas de otros países. 


\section{Referencias}

Comisión Nacional para el Desarrollo de los Pueblos Indígenas (CDI). (2015). Programa Turismo Alternativo en Zonas Indígenas. Recuperado de http://www.cdi.gob.mx/ focalizada/ptazi/index.php

Díaz, F. (2005). Comunidad y Comunalidad. Diálogos en la acción, segunda etapa 2004. Recuperado de http://rusredire.lautre.net/wpcontent/uploads/Comunidad.-y-Ocomunalidad.pdf

Guerrero 0., A. (2015). La comunalidad como herramienta: una metáfora espiral II. Bajo el Volcán, 15(23), 113-129.

Kiss, A. (2004). Is community-based ecotourism a good use of biodiversity conservation fonds? Trends in Ecology \& Evolution. Elsevier, 19(5), 232-237.

Laverack, G. y Thangphet, S. (2007). Building community capacity for locally managed ecotourism in Northern Thailand. Community Development Journal, 44(2), 172-185.

Martínez L., J. (2003). Comunalidad y desarrollo. México: DGCP/Centro de Apoyo al Movimiento Popular Oaxaqueño, A.C.
Niño G., N. S. y Saldaña A., J. (2014). Cíntora, Guanajuato: Geoturismo y Desarrollo Sustentable. En N.S. Niño (coord.), Patrimonio cultural y natural de Iberoámerica (21). México: Ediciones Eon.

Organización Internacional del Trabajo (OIT)Organización Mundial del Turismo (UNWTO). (2014). Panorama OMT del turismo internacional. World Tourism Organization.

Secretaría de Turismo (Sectur). (2015). Datatur. Análisis Integral del Turismo. México. Recuperado de http://www.datatur.sectur.gob.mx/SitePages/ InventarioTuristico.aspx

Secretaría de Turismo (Sectur). (2017). Resultados de actividad turística. Marzo 2017. Recuperado http://www.datatur.sectur.gob.mx/ RAT/RAT-2017-03(ES).pdf

Velázquez S., R. M., Gómez G., J., Gómez V., J., y Pérez R., D. M. (2016). Factores de microinnovación en el ecoturismo indígena de México. Revista Iberoamericana de Contaduría, Economía y Administración, 5(9), 129-147.

Velázquez S., R., y Gómez, J. (2017). Cosmovisión y sustentabilidad comunitaria en la práctica turística. Oaxaca: TECCIS.

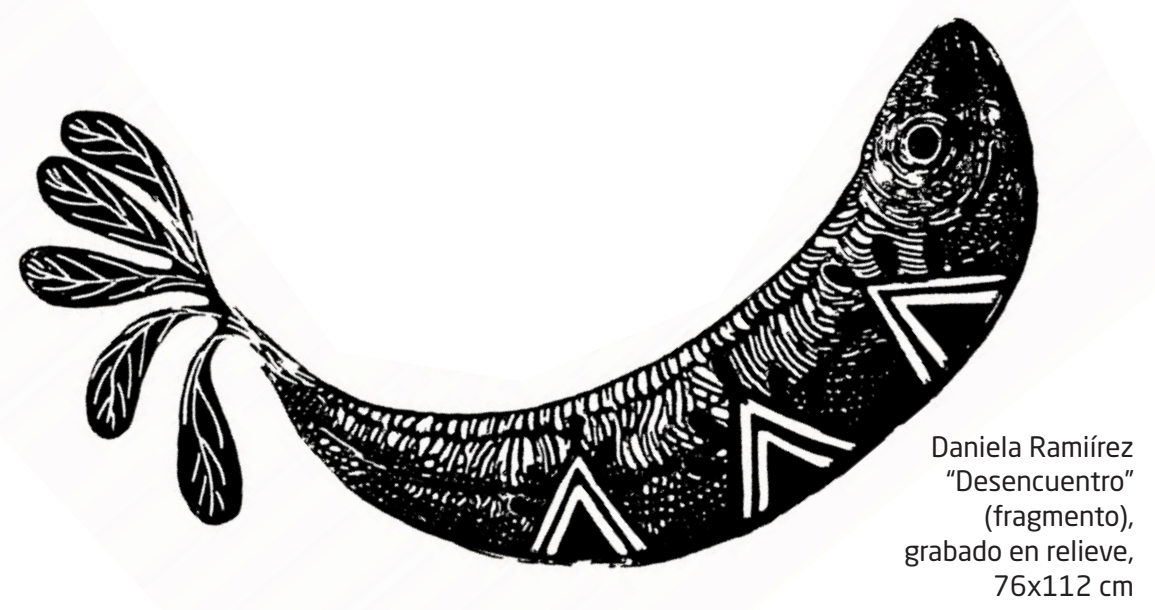




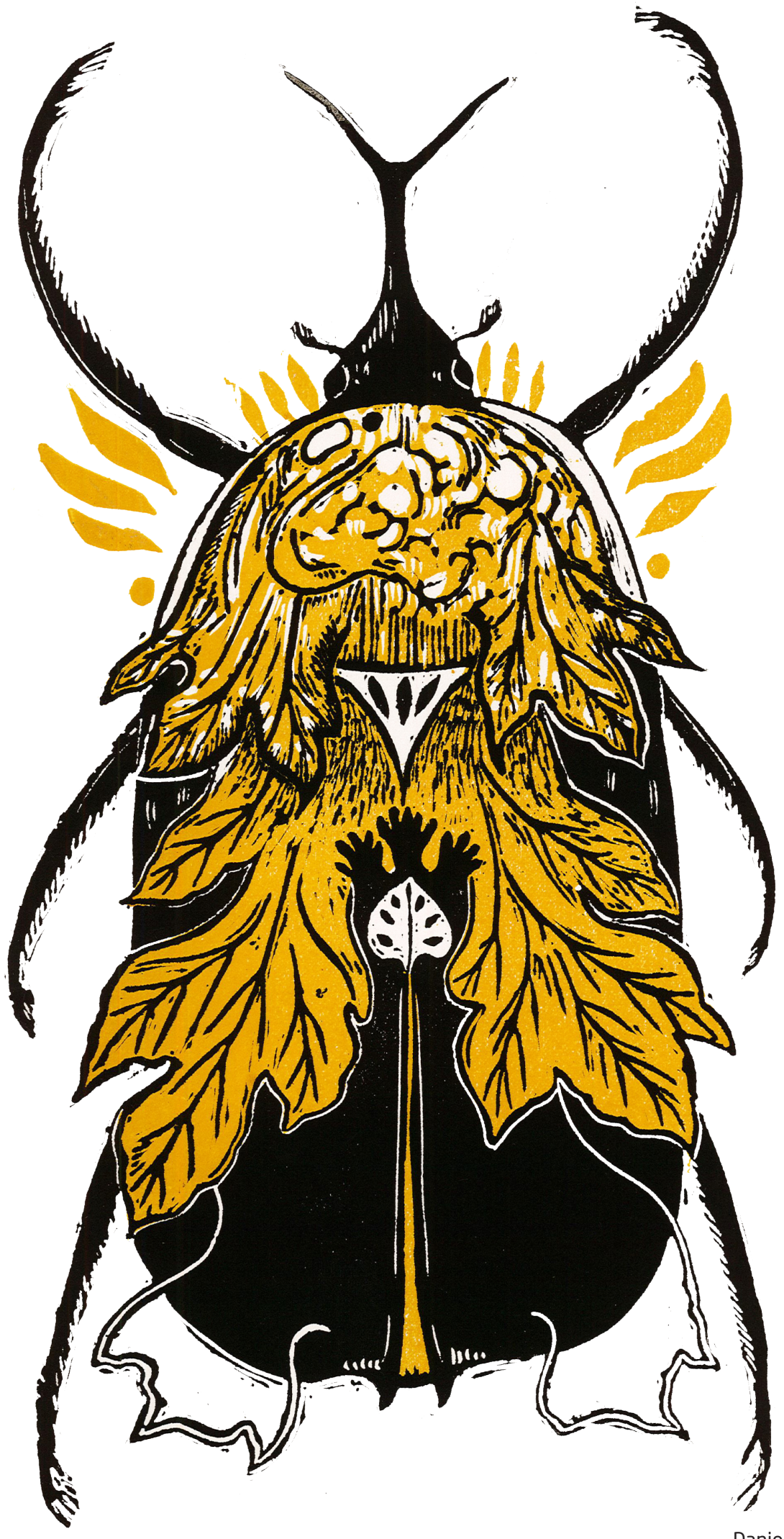

Daniela Ramírez,

"Alevosía III",

linóleo

$50 \times 35 \mathrm{~cm}$ 